\title{
Model of Image Color Difference and Partial Based On RGB Color Distribution Measuring
}

\author{
Weilong Zhou and Chuanlai Yuan * \\ *Address correspondence to Chuanlai Yuan; Email: chuanlai1972@163.com \\ College of Electrical and Information Engineering, Hunan University \\ of Technology, Zhuzhou, 412007, PR China
}

\begin{abstract}
According to the color difference fabric dyeing measurement in production, partial color existed bad real-time problems. Color difference and color of RGB fabric images put forward by using the method of color distribution measurement of general, which needed rapid measurement of large area color. According to RGB distribution histogram of the image, the measured color with sample color similarity and distribution curve of characteristic value was calculated, obtained the RGB color model, and gives a method of a partial color judging. The experiment proves the feasibility of this measurement fabric color difference and the judgment method and effectiveness of partial color. Color cast detection method has overcome the limitations of the traditional methods of detecting image color cast. In order to partial color image detection is performed after correction, using a combination of gray world and a perfect reflection of the color correction method, to make up for the shortcomings of traditional method. The experimental results show that, the color deviation correction method of image analysis based on detection improved the reliability of color cast detection. The method uses characteristics of image analysis, it has universal applicability.
\end{abstract}

Keywords: Histogram equalization; Image process; Affinity fitness function; Color correction

\section{Introduction}

In the judgment of the fabric dyeing color difference and color offset production, at present the general artificial color detection and color measurement of two kinds of methods of color measurement [1]. Artificial color detection rely on visual observation identification, observation results are influenced by the individual differences and experience and other factors, can only do a rough estimate of the chromatic aberration is unable to obtain quantitative results, the partial color judgment is entirely dependent on the observer's experience, so the color accuracy is not high, mainly used in the dyeing production site; color measurement instrument is measured by measuring the color of fabric surface on the visible light reflectance $(380 \mathrm{~nm} 780 \mathrm{~nm})$, the $\mathrm{XYZ}$ value in getting all the reflectance spectra of color three stimulation, according to the obtained the measured color difference fabrics and fabric sample fabric and partial color difference formula and hue formula [2]. Color measurement results are accurate color measurement instrument, but only for a small range (color measurement instrument window) in color measurement, color measurement can not realize the large area, also asked the fabric smooth surface drying [3].

By photographic method for measuring color and partial color, there have been few studies related. In the color measurement, $\mathrm{Li} \mathrm{Wu}$ use of color space conversion method for camera image RGB color values into chroma values [4]. Ye Guojun et al used Lab color information of image retrieval [5]. In the color cast detection, Xu Xiaoshao [6] uses each pixel in an image of color values, calculate all pixels of the center of gravity to 
determine chroma, color partial image; Zheng Jianhua according to the intensity difference between color images of the distribution curve of RGB value, to determine the color of partial color. The shortcomings of these methods are not considered photographic error nonlinear photographic equipment brought by the response of the reaction of the human eye to partial color is actually based on the color after the mixing effect [7].

Methods RGB distribution curve detection color and color through the proposed in this paper, is to get the distribution curve of samples tested their color and color, through the curve feature values and distribution characteristics, proposed color decision model, and gives a method of partial color judgment [8-12]. The innovation goods images were obtained with photography, and measuring color aberration and partial color through RGB image histogram distribution difference [13, 14]. In order to have general applicability, this paper does not make any ideal hypothesis, given only partial color image in the situation, through carries on the automatic detection and correction to get the or estimates original unbiased color images. The algorithm firstly by color histogram of partial color images were analyzed, and then the discriminant partial color, partial color channel, and partial color type, and treated respectively.

\section{Related Knowledge Overview}

\subsection{Image Preprocessing Method}

Each pixel of the color image is usually represented by 3 bytes, each byte corresponds to the RGB component of the brightness, and a pixel gray level image transformed by a byte to represent, the little gray value greater brightness is bright; smaller values, brightness is black. Conversion between the used formula [15-18]:

$$
\text { Grey }(i, j)=0.1 * r(i, j)+0.59 * g(z, j)+0.3 * b(i, j)
$$

The input value of gray level higher than the compressed into narrow output image in lighter gray level, the output is high contrast images:

$$
S=T(r)=\frac{1}{1+(m / r)^{E}}
$$

According to the formula, Histogram equalization $(\mathrm{x}, \mathrm{x})$ on the input gray level executive changes the output gray level $\mathrm{S}$.

$$
s=T(r)=\int_{0}^{r} p_{r}(\omega) d \omega
$$

In the formula $(\mathrm{w})$ representation of the probability density function of the gray level in a given image, $w$ is the 11 sub variable integral. You can get the probability density function of the output gray level is homogeneous [19]:

$$
p_{s}(s)=\left\{\begin{array}{l}
1,0 \leq s \leq 1 \\
0, \text { else }
\end{array}\right.
$$

The filtering process is the first two-dimensional sliding template selection of specific structure, and then will be arranged according to the size of pixel values in the form template, the data sequence monotone. The output formula of two dimensional median for:

$$
G(x, y)=\operatorname{Mid} f(x-i, y-j),(i, j)
$$

Gaussian smoothing filter is a widely used algorithm in image processing, mathematical expressions for Gaussian smoothing filter:

$$
\begin{gathered}
f^{\prime}(x, y)=f(x, y) * g(x, y) \\
g(x, y)=\frac{1}{\sqrt{2 \pi \sigma}} \exp \left(-\frac{x^{2}+y^{2}}{2 \sigma^{2}}\right)
\end{gathered}
$$


Let $\mathrm{X}$ and $\mathrm{B}$ is the set of integer space in the $\mathrm{Z}$, where Ais the original image, and the $\mathrm{B}$ structure element.

a) $\mathrm{B}$ on the expansion of Aoperations is defined as:

$$
A \oplus B=\left\{z \mid(B)_{z} \cap A \neq \varnothing\right\}
$$

b) Corrosion of $\mathrm{B}$ on $\mathrm{A}$ to define operations:

$$
A \circ B=(A \Theta B) \oplus B
$$

c) $\mathrm{B}$ on $\mathrm{A}$ open operation is defined as:

$$
A \Theta B=\left\{z \mid(B)_{z} \subseteq A\right\}
$$

\subsection{Partial Color of Traditional Detection Methods}

In order to reproduce the real color must influence the color temperature of the light source so as to remove the environment factors, namely the elimination of partial color. However, a prerequisite for removing image color deviation is to detect the presence of partial color and color deviation degree image. At present, the research of color cast detection has made some progress, the detection method which is more representative of the color cast, including histogram statistics [19], gray balance method [20], white balance method [21-23].

White balance method is for the images have reflection, that high light reflection or white space can reflect the light reflective chromaticity, maximum brightness statistics for the three channels, the color space transformation, to obtain the Lab coordinate relatively uniform, and the ideal light source color distance calculation, so as to determine the existence of partial color. But when there is not the object of white or high light shooting, color cast detection result is distortion.

To measure the two color similar items color difference and partial color (partial color refers to bias red/green/blue color, if can judge the partial color can adjust the production process so that the production is not partial color, objects are solid colors, such as pale green or pinkish red, there is no pattern, now general approach is to use the color measurement instrument, real-time differential:

With getting the color of XYZ three stimulate value, in the use of standard illuminant conditions, the convert relation between $X Y Z$ three stimulated value:

$$
\left\{\begin{array}{l}
L=116 f(Y / 100)-16 \\
a=500[(f(X / 94.825)-f(Y / 100)] \\
b=200[f(Y / 100)-f(Z / 107.381)]
\end{array}\right.
$$

Interpretation of partial color such as the common blue, there is dark blue, light blue, blue partial red, blue greenish etc, by measuring $\mathrm{H}$ tone can be measured with color measurement instrument.

\section{Histogram of Partial Color Image in RGB Space Analysis}

\subsection{Color Histogram Statistics}

The overall color histogram statistics can often give the image performance. Figure 1 shows a partial color image, the color histogram statistics. Obviously, according to the average brightness R, G, B channel can estimate whether partial color image. The brightness of $\mathrm{R}$ component values are higher, according to $\mathrm{R}, \mathrm{G}, \mathrm{B}$ color theory, the whole image of partial red, and this is consistent with the subjective feeling. But for a variety of image in different applications, histogram statistics is difficult to obtain comprehensive, accurate judgments. 

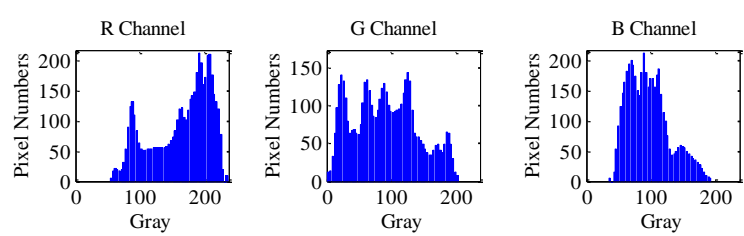

(a) Unsaturated partial color
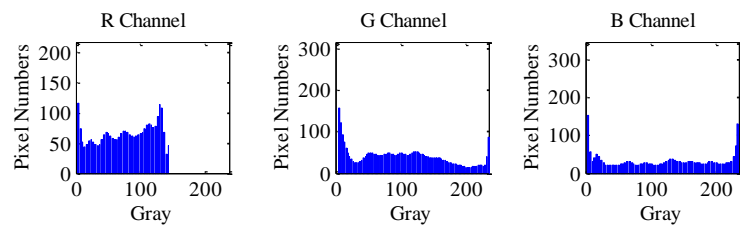

(b) Saturated partial color

Figure 1. The Color Histogram Statistics

Balance method is targeted to meet gray world assumptions, namely the whole image of the R, G, B of equal mean, embodied in the neutral grey, average luminance statistics for the 3 channels, the color space transformation, to obtain the Lab coordinates of relatively homogeneous, calculation and neutral point color distance, so as to determine the existence of partial color. But when the environment is too bright or too dark, or the color of the image is relatively single, gray balance method almost completely failure, its basic reason is no longer meet the gray world assumption on this basic premise.

Figure 1 (a) first cases belong to the partial color, gray histogram of RGB from three channels, in addition to a saturation phenomenon of mild $R$ channel image, all pixels in $G$ and $\mathrm{B}$ channel image did not reach saturation, all pixels in the basic channel image are within the dynamic range of the camera photosensitive strength. Figure 1 is visible, due to the $\mathrm{R}$ channel image gray value is the number of pixels above 200 is far greater than the number of pixels corresponding to a gray value of $\mathrm{G}$ and the image of the $\mathrm{B}$ channel, and the gray value greater than $G$ and $B$ channel in $R$ channel most pixel gray value, therefore the $\mathrm{R}$ channel is a serious deviation color channel. Then the same comparison between $\mathrm{G}$ and $\mathrm{B}$ channel, $\mathrm{G}$ channel is apparently slightly color channel, denoted as $\mathrm{R}>\mathrm{G}>\mathrm{B}$.

\subsection{Establishment of Color Image Model}

In this paper, a total of 10 images (Figure 2) obtained from the experiment group ( $\mathrm{n}=$ 2), each group in terms of color is very easy to distinguish.

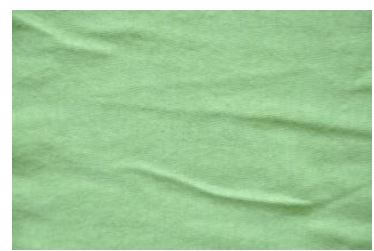

$(1-\mathrm{a})$

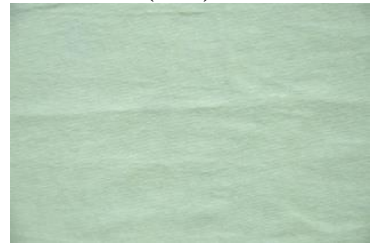

(2-a)

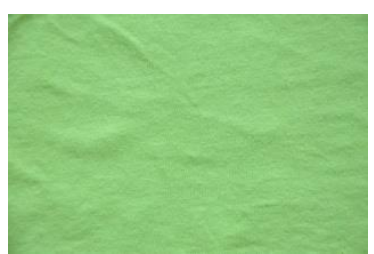

(1-b)

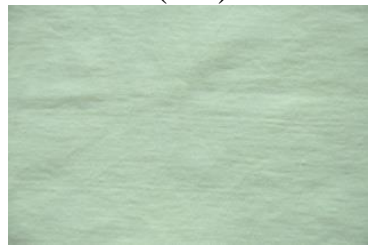

(2-b) 


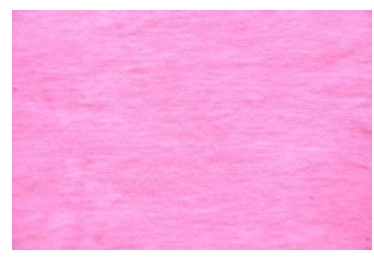

(3-a)



$(4-a)$

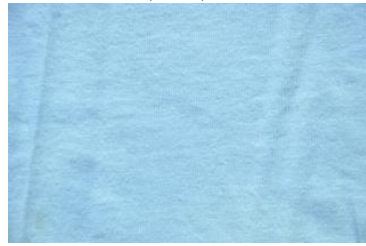

$(5-a)$

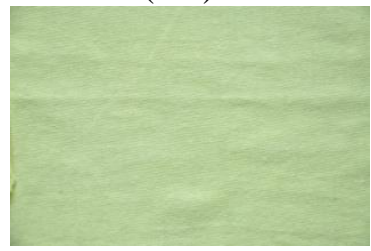

(6-a)

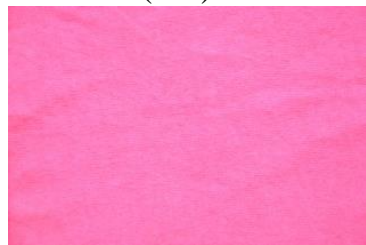

$(7-a)$

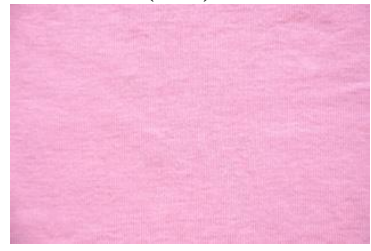

$(8-\mathrm{a})$

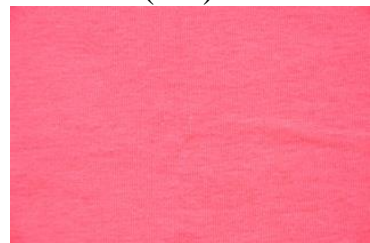

(9-a)

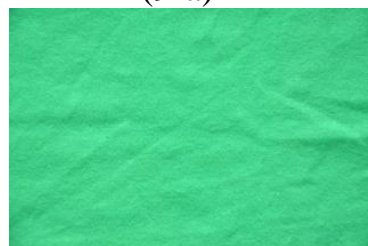

$(10-a)$

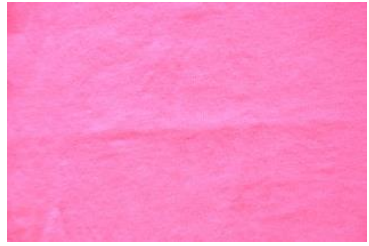

(3-b)

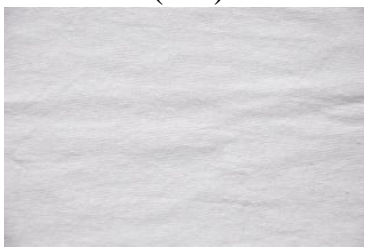

(4-b)

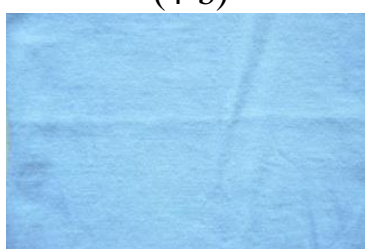

$(5-b)$

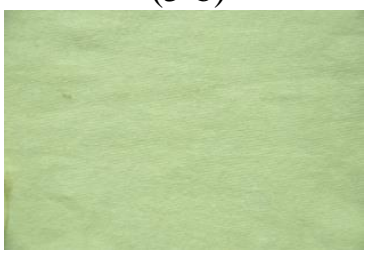

$(6-b)$

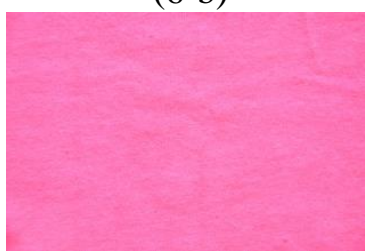

(7-b)

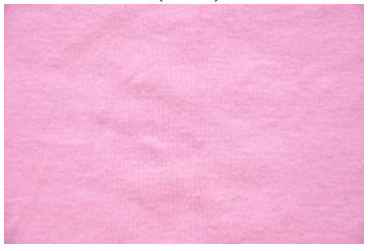

$(8-b)$

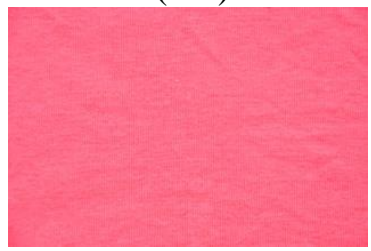

(9-b)

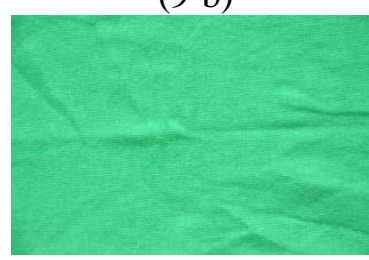

(10-b)

Figure 2. The Experiment Sample Images 


\subsection{Methods RGB Image Distribution Map}

Image drawing method of RGB distribution map in matlab: image=imread("filel.....image.jpg);

$$
\mathrm{R}=\text { image }(:,:, 1) ; \mathrm{G}=\text { image }(:,:, 2) ; \mathrm{B}=\text { image }(:,:, 3) \text {; }
$$

$\% \% \%$ plot curve :

$\mathrm{x}=0: 255$ (\% color range $=0: 255)$

plot(x,imhist $(\mathrm{R}))$, G.B are the same

Linear scanner signal, also known as the grey balance correction, the scanner the processed signal is a linear function of brightness. Remember the gray balance after correction for scanner signals $(\mathrm{R} 1, \mathrm{G} 1, \mathrm{~B} 1)$, then:

$$
R_{1}=g_{R}(R), G_{1}=g_{G}(G), B_{1}=g_{B}(B)
$$

Type $\mathrm{g}_{\mathrm{R}}, \mathrm{g}_{\mathrm{G}}, \mathrm{g}_{\mathrm{B}}$ are linear function $\mathrm{R}, \mathrm{G}, \mathrm{B}$ channel.

\section{Results and Simulation}

\subsection{Algorithm of Automatic Detection and Correction of Partial Color Image}

Through the analysis of the above two kinds of partial color, can be partial color detection and correction algorithm is proposed to combine the structure, as shown in Figure 3.

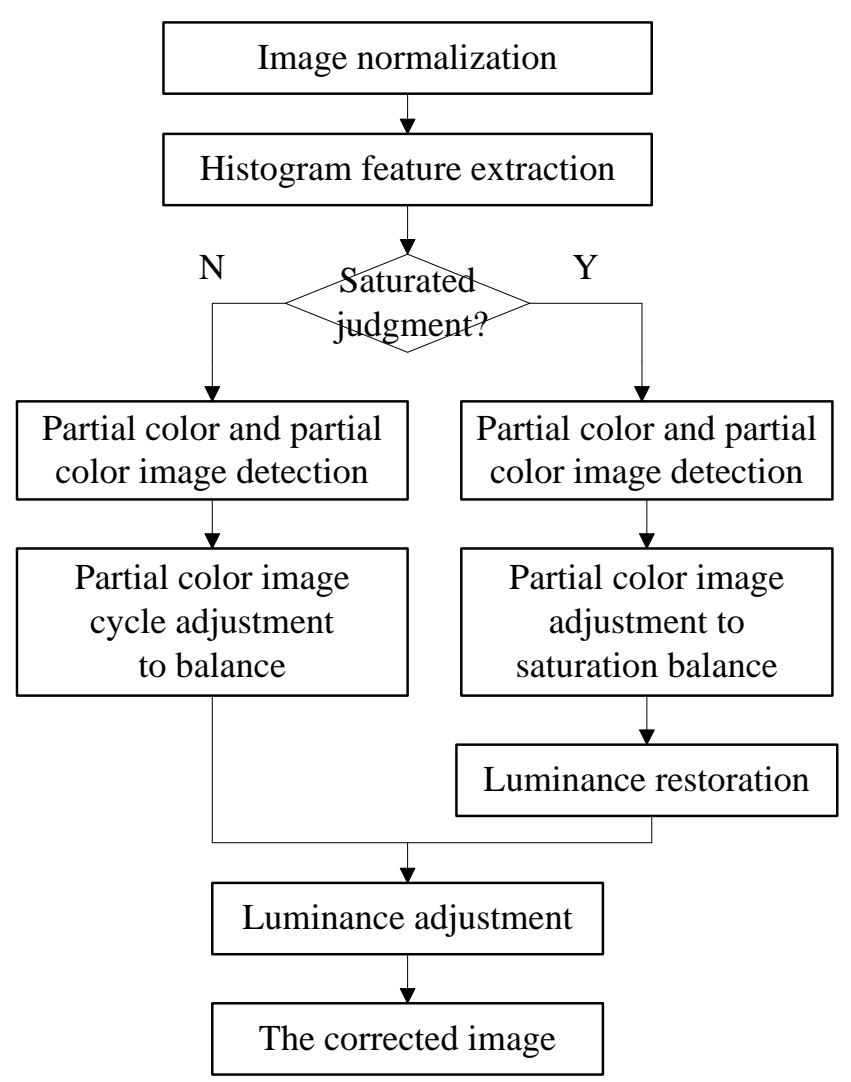

Figure 3. The Correction Algorithm Proposed to Combine the Structure

The input image is normalized, because all kinds of gray histogram which can in the retaining ring like speed processing detail features conditions then extract $R, G, B$ channel image, such as the maximum and minimum values of each channel of the image gray 
scale, for the number of pixels on each channel specific gray and the gray of the image whether to achieve positive saturation characteristics.

\subsection{RGB Histogram Distribution Curve}

In this paper, RGB distribution curves of the experimental image are as follows, and there are differences between each other. Red-green-blue respectively for R-G-B distribution in the fig, the solid line as the sample image on the left, dashed lines as the measured object image on the right.
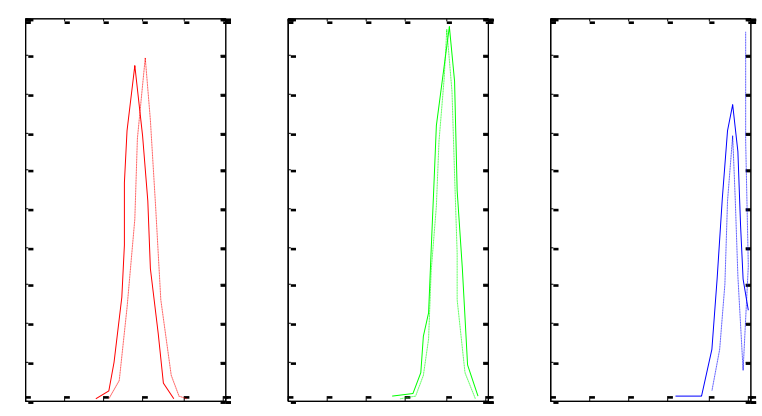

\section{Figure 4. RGB Histogram Distribution Curve}

RGB histogram distribution curve was shown in Figure 4. According to the distribution curve of the obtained data, respectively is the mean of $R$, variance, skewness, followed by $\mathrm{G}$ and $\mathrm{B}$. In order to understand the image of partial color, choose R, G, B histogram 3 color channel image and the color space transformation is obtained after the luminance channel gray histogram of image is divided into partial color. Figure 4 was two kinds of partial color image typical. Figure 4 are respectively the gray histogram in RGB space of the image.

\subsection{Experimental Verification}

Color difference value $\mathrm{DE}=9.67$ bigger than the specified value 3.0, visible color difference too big fail. In it $\mathrm{Dl}=-8.13, \mathrm{Da}=0.97, \mathrm{Db}=-5.14$. Sample color - tested color. $\mathrm{DE}=\operatorname{sqrt}\left(\mathrm{Dl}^{\wedge} 2+\mathrm{Da}^{\wedge} 2+\mathrm{Db}^{\wedge} 2\right)$ There are color difference $\triangle \mathrm{E}=9.67$ (among $\triangle \mathrm{L}=8.13$, $\triangle \mathrm{a}=0.97, \triangle \mathrm{b}=5.14$ ) between two items with the color measurement instrument. The physical map color is dark red relative to the sample map color. 


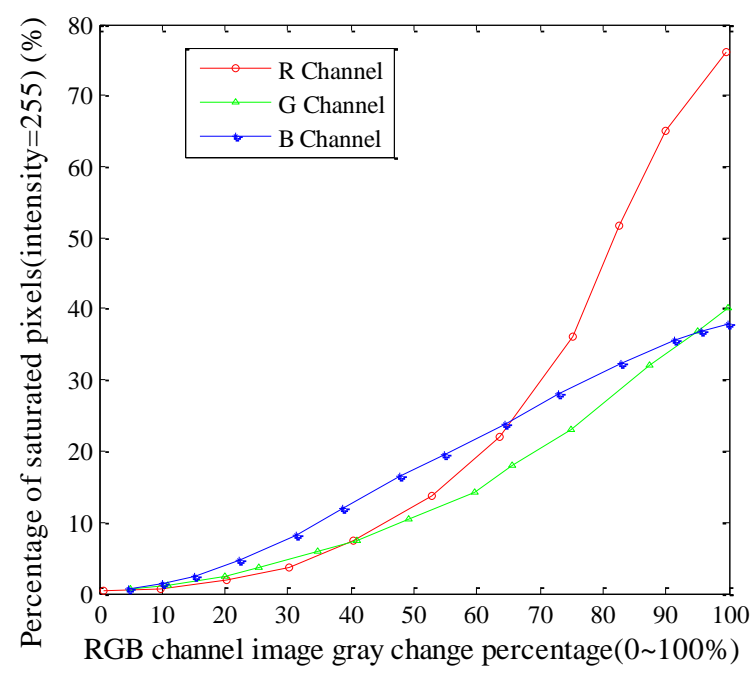

\section{Figure 5. The Diagram in R, G, B 3 Channel Pixel Gray}

The diagram in Figure 5 shows the diagram in R, G, B channels pixel gray occurred respectively positive change, change the channel image saturation pixel number proportions. By the visible, initially, each channel image without saturation, when the channel image pixel gray positive increases, then a saturated pixel proportional growth speed gradually accelerated.

\section{Conclusion}

RGB each channel image saturation pixel ratio with the channel image gray growth rate curve. In this paper, the characteristic of partial color image itself is analyzed, so it has universal applicability. Experiments show that, in the partial color greatly, this algorithm can obtain satisfactory results. If the algorithm is applied to the skin recognition, face detection, object tracking algorithm before, used as a processor, no doubt the algorithm can greatly enhance the applicability and performance of the proposed algorithm.

\section{Acknowledgement}

This work was supported by the Hunan Provincial Natural Science Foundation of China (Grant No. 2015JJ5025).

\section{References}

[1] Li Jian, Pan Qing, Yang Tian. "Color Based Grayscale-fused Image Enhancement Algorithm for Video Surveillance", In Proceeding of the third international conference on image and graphics, pp. 47-50, 2004.

[2] T. Matsuo, Y. Kaneko, M. Matano, "Introduction of Intelligent Vehicle Detection Sensors", IEEE/IEEJ/JSAI International Conference on Intelligent Transportation Systems, vol. 5, no. 12, pp. 709-713, 1999.

[3] Adrian Perrig, John Stankovic, David Wagner, "Security in Wireless Sensor Network," ACM, vo. 47, no. 6, pp. 53-57, 2004.

[4] J. Deng, R. Han, S. Mishra, "Inrusion tolerance and Anti-traffic Analysis Strategies in Wireless Sensor Networks," IEEE 2004 International Conference on Dependable Systems and Networks, vo. 10, no. 11, pp. 477-490, 2004.

[5] WangJue, WangRen, MiaoDuoqian, "Data enriching based on rough set theory", Journal of Environmental Sciences, vol. 29, no. 3, pp. 63-69, 2010. 
[6] HouChangjun, LiJiangjie, DanqunHuo, Xiao gang Luo, Jiale Dong, Mei Yang, Xiao Jie Shi, " Design of an embedded gas detector based on spectral analysis ", Chinese Journal of Scientific Instrument, vol. 29, no. 4, pp. 471-475, 2008.

[7] Yang H, Shao L, Zheng F, et al. Recent advances and trends in visual tracking: A review [J]. Neurocomputing, 2011, 74(18): 3823-3831.

[8] Zhang S, Yao H, Sun X, et al. Sparse coding based visual tracking: Review and experimental comparison [J]. Pattern Recognition, 2013, 46(7): 1772-1788.

[9] Cannons K. A review of visual tracking [J]. Dept. Comput. Sci. Eng., York Univ., Toronto, Canada, Tech. Rep. CSE-2008-07, 2008

[10] Borishansky, V.M. Heat transfer to a liquid flowing over surface heated to a temperature above the boiling point in Problems of heat transfer During a Change State (A college of articles),United States Atomic Energy Commission Translation Service, Washinglon,USA p3405-3445.

[11] Hayder Salman Marios Soteriou, Lagrangian simulation of evaporating droplet sprays ,American Insititute of Phsics .2004(12),p4601-4622.

[12] Chang C, Ansari R. Kernel particle filter for visual tracking [J]. Signal processing letters, IEEE, 2005, 12(3): 242-245.

[13] Grabner H, Bischof H. On-line boosting and vision[C]. Computer Vision and Pattern Recognition, 2006 IEEE Computer Society Conference on. IEEE, 2006, 1: 260-267.

[14] Grabner H, Leistner C, Bischof H. Semi-supervised on-line boosting for robust tracking [M]. Computer Vision-ECCV 2008. Springer Berlin Heidelberg, 2008: 234-247.

[15] Stalder S, Grabner H, Van Gool L. Beyond semi-supervised tracking: Tracking should be as simple as detection, but not simpler than recognition[C]. Computer Vision Workshops (ICCV Workshops), 2009 IEEE 12th International Conference on. IEEE, 2009: 1409-1416.

[16] Babenko B, Yang M H, Belongie S. Visual tracking with online multiple instance learning [C]. Computer Vision and Pattern Recognition, 2009. CVPR 2009. IEEE Conference on. IEEE, 2009: 983 990.

[17] Cortes C, Vapnik V. Support-vector networks [J]. Machine learning, 1995, 20(3): 273-297.

[18] Lowe D G. Distinctive image features from scale-invariant keypoints[J]. International journal of computer vision, 2004,60(2): 91-110.

[19] Dalai N,Triggs B Histograms of oriented gradients for human detection[C].Computer Vision and Pattern Recognition, 2005. CVPR 2005. IEEE Computer Society Conference on. IEEE, 2005, 1: 886893.

[20] Dalai N,Triggs B,Schmid C. Human detection using oriented histograms of flow and appearance [M], Computer Vision-ECCV 2006. Springer Berlin Heidelberg,2006: 428-441.

[21] Chen Q, Georganas N D,Petriu E M. Real-time vision-based hand gesture recognition using haar-like features [C].Instrumentation and Measurement Technology Conference Proceedings, 2007. IMTC 2007.IEEE. IEEE, 2007: 1-6.

[22] Xiang Y,Su G. Multi-parts and multi-feature fusion in face verification[C].Computer Vision and Pattern Recognition Workshops, 2008. CVPRW 08. IEEE Computer Society Conference on IEEE, 2008: 1-6.

[23] Chen J, He Y,Wang J. Multi-feature fusion based fast video flame detection [J], Building and Environment, 2010,45(5): 1113-1122.

\section{Authors}

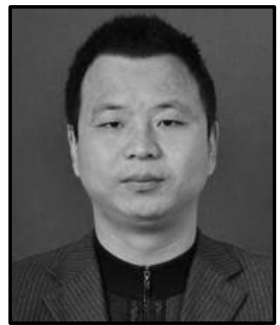

Weilong Zhou, He received his master's degree in 2011 from Hunan University, China. Currently, he works as a lecturer at College of Electrical and Information Engineering, Hunan University of Technology. His current research interests include embedded system design and application, wireless sensor networks technology and application.

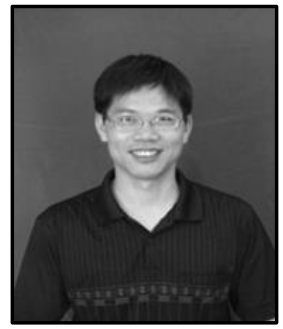

Chuanlai Yuan, He received his bachelor degree in 1995 from Hunan Agricultural University, China. Currently, he works as associate professor at College of Electrical and Information Engineering, Hunan University of Technology. His current research interests include automatic detection, optimal control and design and control of industry robot. 
International Journal of Grid and Distributed Computing Vol. 9, No. 8 (2016) 